\title{
Entwurfskonzept einer Car2Car-Multiband-Dachantenne
}

\author{
Y. Wang, M. Reit, and W. Mathis \\ Institut für Theoretische Elektrotechnik, Leibniz Universität Hannover, Appelstr. 9A, 30167 Hannover, Germany \\ Correspondence to: Y. Wang (ywang@ @et.uni-hannover.de)
}

\begin{abstract}
Due to the vastly increasing use of wireless services in the car, such as WiFi, Car2Car and LTE, the requirements on bandwidth and radiation pattern of the roof antenna systems become more challenging. In this work, a design concept for multi-band roof antenna systems is presented. We aim to get a higher bandwidth and an almost circular radiation pattern on the horizontal plane. Moreover, the antenna length is considered in order to fulfill the requirements set by construction ECE-regulations (ECE, 2010). The applicability of the design concept is not limited to multi-band roof antennas, it can also be used for a general wideband antenna design. For illustration of this concept, a multi-band roof antenna with a bandwidth of $780 \mathrm{MHz}$ to $5.9 \mathrm{GHz}$ and a near circular radiation pattern with an average gain of $G=3 \mathrm{dBi}$ (at $5.9 \mathrm{GHz}$ ) on the horizontal plane is designed. The simulation and measurement results are presented.
\end{abstract}

Zusammenfassung. Aufgrund der immer umfangreicheren Nutzung neuer Funkdienste im KFZ, wie WLAN, Car2Car und LTE, steigen die Anforderungen bezüglich der Bandbreite und der Strahlungscharakteristik der verwendeten Antennensysteme stetig an. In dieser Arbeit wird ein Entwurfskonzept für eine Car2Car-Multiband-Dachantenne vorgestellt. Ziele bei einem solchen Entwurf sind eine möglichst hohe Bandbreite sowie ein in horizontaler Ebene hoher und gleichmäßig verteilter Antennengewinn. Hierbei muss die amtliche Vorgabe (ECE, 2010) für die Antennenhöhe berücksichtigt werden. Die Anwendbarkeit des Verfahrens beschränkt sich nicht nur auf Multiband-Dachantennen, sondern kann ebenso für den allgemeinen Breitbandantennenentwurf genutzt werden. Als Beispielanwendung wird mit dem hier vorgestellten Konzept eine Multiband-Dachantenne mit einer Bandbreite von $780 \mathrm{MHz}$ bis $5,9 \mathrm{GHz}$ und einer nahezu runden Strahlungscharakteristik sowie einem durchschnittlichen Antennengewinn von $G=3 \mathrm{dBi}$ (bei $5,9 \mathrm{GHz}$, in horizontaler Ebene) entworfen. Zusätzlich werden die Simulations- und Messergebnisse vorgestellt.
Tabelle 1. Die aktuellen Funkdienste im KFZ mit entsprechenden Frequenzen.

\begin{tabular}{llllll}
\hline & $\begin{array}{l}\text { LTE } \\
\text { low }\end{array}$ & $\begin{array}{l}\text { GSM } \\
900\end{array}$ & UMTS & $\begin{array}{l}\text { WiFi } \\
\text { /WLAN }\end{array}$ & $\begin{array}{l}\text { WiMAX } \\
\text { low }\end{array}$ \\
\hline$f_{\min }$ & 780 & 810 & 1710 & 2400 & 2495 \\
$f_{\max }$ & 860 & 960 & 2170 & 2485 & 2690 \\
\hline & LTE & WiMAX & WiFi & WiFi & C2C \\
& high & high & /WLAN & $/$ WLAN & /DSRC \\
\hline$f_{\min }$ & 2500 & 3300 & 5150 & 5470 & 5850 \\
$f_{\max }$ & 2690 & 3800 & 5350 & 5825 & 5925 \\
\hline
\end{tabular}

\section{Introduction}

Die Car2Car-Kommunikation hat eine große Bedeutung für die Fahrsicherheit (Lübke, 2004). Im Jahr 2008 wurde der Frequenzbereich von $5875 \mathrm{MHz}$ bis $5905 \mathrm{MHz}$ mit einer spektralen EIRP-Dichte von $23 \mathrm{dBm} / \mathrm{MHz}$ und einer maximalen gesamten Sendeleistung von $33 \mathrm{dBm}$ für sicherheitsbezogene Anwendungen intelligenter Verkehrssysteme (ITS) in Europa freigegeben (EU, 2008). In Bezug auf die aktuellen Funkdienste, arbeitet ein modernes Funkkommunikationssystem im Auto in einem Frequenzbereich von $780 \mathrm{MHz}$ bis $5,925 \mathrm{GHz}$. Es umfasst damit sechs unterschiedliche Funkdienste (vgl. Tabelle 1). Hierdurch werden besondere Anforderungen an die Richtcharakteristik der Car2Car-Antenne gestellt. Da sich die Verkehrsteilnehmer in der Praxis in der horizontalen Ebene befinden, soll auf dem Horizontaldia$\operatorname{gramm}\left(\vartheta=90^{\circ}\right)$ bei $5,9 \mathrm{GHz}$ keine benachteiligte Richtung vorhanden und der Antennengewinn möglichst groß sein. Ferner ist vorgeschrieben, dass die maximale Höhe der Antenne, einschließlich ihres Gehäuses, nicht mehr als $70 \mathrm{~mm}$ 
betragen darf, wenn die Antenne im hinteren Bereich des Fahrzeugdachs montiert wird (ECE, 2010). Zurzeit existiert noch keine, im industriellen Maßstab produzierte, Autodachantenne, welche sowohl für die Car2Car-Anwendung geeignet ist, als auch alle geforderten Funkdienste unterstützt. Für die oben genannten Anforderungen wurde in dieser Arbeit eine Car2Car-Multiband-Dachantenne, im Folgenden als Multiband-Antenne bezeichnet, entworfen. Das Verfahren wird im Folgenden detailliert vorgestellt und mit einem Vergleich zwischen den Simulations- und Messergebnissen verifiziert.

\section{Entwurfskonzept}

Für den Entwurf der Multiband-Antenne werden die folgenden sechs Entwurfsschritte in gegebener Reihenfolge durchlaufen.

\section{Zielsetzung}

2. Auswahl der Antennen-Basisstruktur

3. Grunduntersuchung der ausgewählten Antennentypen

4. Entwurf und Optimierung

5. Fertigung

6. Messung

\subsection{Zielsetzung}

Wie in der Einleitung bereits erwähnt wurde, soll die Multiband-Antenne folgende Anforderungen erfüllen:

- Die Eingangsimpedanz der Antenne muss von $780 \mathrm{MHz}$ bis $5,925 \mathrm{GHz}$ breitbandig für einen $50 \Omega$ Koaxialanschluss angepasst werden.

- Das Horizontaldiagramm $\left(\vartheta=90^{\circ}\right)$ der Antenne soll bei $5,9 \mathrm{GHz}$ möglichst rund und der Antennengewinn möglichst groß sein.

- Die Konstruktion der Antenne muss kompakt und robust sein. Die Antennenhöhe soll $70 \mathrm{~mm}$ nicht überschreiten.

\subsection{Auswahl der Antennen-Basisstruktur}

Zur Erfüllung der bei der Zielsetzung festgelegten Anforderungen werden entsprechende Antennen-Basisstrukturen ausgewählt. Um eine breitbandige Impedanzanpassung von $780 \mathrm{MHz}$ bis $5,925 \mathrm{GHz}$ gewährleisten zu können, fällt die Wahl auf eine Breitband-Antennenstruktur, wie z. B. die Bow-Tie- oder die Cone-Antenne (Balanis, 2005). Wegen

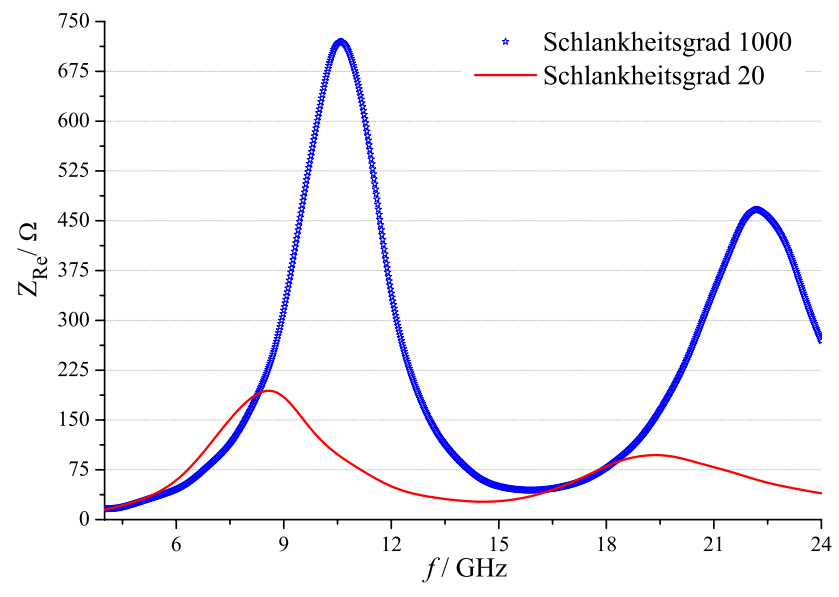

Abbildung 1. Realteil der Eingangsimpedanz eines Monopols mit zwei unterschiedlichen Schlankheitsgraden.

des Koaxialanschlusses sind die asymmetrischen Antennenstrukturen von besonderem Interesse. Ein vertikal polarisierter Strahler ist bei korrekter Bemessung eine ausgezeichnete Car2Car-Antenne, da er eine horizontale Rundcharakteristik besitzt. Diese horizontale Rundcharakteristik bietet die Gewähr, dass keine Richtung benachteiligt wird. Es werden daher vertikal polarisierte Strahler unterschiedlichen Typs ausgewählt. Da allein eine $\lambda / 4$-Monopolantenne für das GSM-Band bereits eine Höhe von über $80 \mathrm{~mm}$ besitzt, wird eine Monopolantenne mit Dachkapazität zur Reduktion der Antennenhöhe in Betracht gezogen (Lange and Löcherer, 1992).

\subsection{Grunduntersuchung der ausgewählten Antennen}

Die gewählten Antennen-Basisstrukturen werden mittels Simulationen untersucht. In diesem Artikel wird exemplarisch das Untersuchungsergebnis einer schmalbandigen MonopolAntenne mit variierten Antennenparametern dargestellt. Die Abb. 1 zeigt den über die Simulationen ermittelten Realteil der Eingangsimpedanz eines zylindrischen Monopols für die Schlankheitsgrade ${ }^{1} 20$ und 1000. Es wird deutlich, dass die Schwankung des Impedanzverlaufs im Frequenzgang bei einem kleinen Schlankheitsgrad deutlich geringer ist (Rothammel and Krischke, 2001). Dies ist eine wichtige Erkenntnis für die Impedanzanpassung. Bei weiteren Simulationen werden die Richtcharakteristik und die Antennengeometrie unter verschiedenen Randbedingungen untersucht. Die hierdurch gewonnen Erkenntnisse werden beim nächsten Schritt Entwurf und Optimierung berücksichtigt.

\footnotetext{
${ }^{1}$ Der Schlankheitsgrad wird durch $l / r$, dem Verhältnis zwischen Länge und Radius, ausgedrückt.
} 


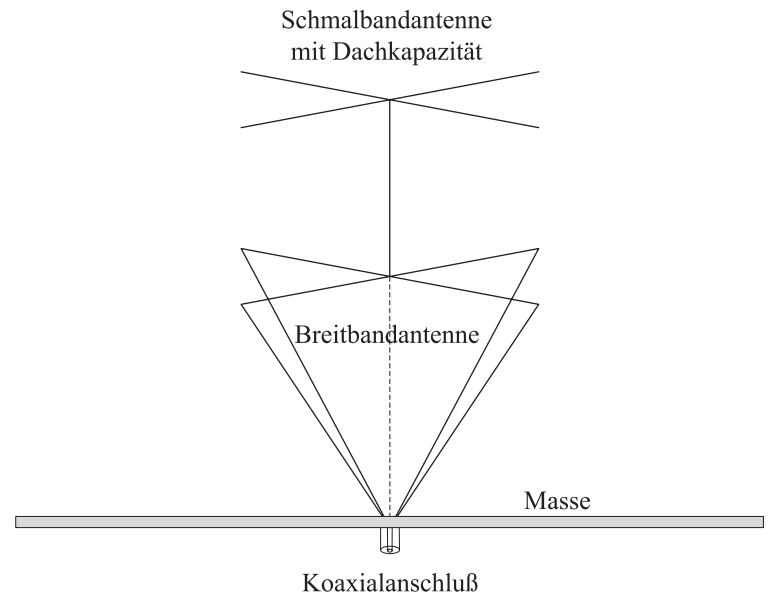

Abbildung 2. Prinzipbild der Multiband-Antenne.

\subsection{Entwurf und Optimierung}

Um die Multiband-Antenne kleinzuhalten, aber gleichzeitig ihr Breitbandverhalten gewährleisten zu können, wird eine Kombination aus einer Breitband- und einer Schmalbandantenne angewendet (vgl. Abb. 2). Die Breitbandantenne ist für den oberen Frequenzbereich zuständig. Wegen der geringeren Abmessungen ist die schmalbandige Monopolantenne für die tiefen Frequenzen, wie z. B. für GSM und LTE low, gut geeignet. Eine Maßnahme um eine Impedanzanpassung gewährleisten zu können, ohne dabei die $70 \mathrm{~mm}$-Grenze zu überschreiten, ist die Nutzung einer Dachkapazität. Die runde Richtcharakteristik für die Car2Car-Anwendung wird mit einer achsensymmetrischen Antennenstruktur erreicht. Mit diesen Kenntnissen wird das erste Antennenmodell entworfen. Auf der Basis dieses ersten Modells werden die Antennenabmessungen unter Berücksichtigung der elektrischen und mechanischen Anforderungen bestimmt. Hierfür werden zunächst gültige Bereiche für die Abmessungen, in denen die Impedanzanpassung erfüllt wird, mittels Simulationen ermittelt. Der dabei angewendete Löser ist der Transient Solver ${ }^{2}$ vom CST Micorwave Studio. Es wird für den Rand der Simuoationsumgebung angenommen, dass sich die EM-Wellen dort wie in Freiraum ausbreiten (Open (add space)). Als Gütemaß einer optimalen Impedanzanpassung wird $\left|S_{11}\right|<-10 \mathrm{~dB}$ für den gesamten Frequenzbereich genommen. Im Hinblick auf den Antennengewinn in der horizontalen Ebene, die minimale Antennenhöhe und die Bandbreite des jeweiligen Funkdienstes, lassen sich die Strukturgrößen in den ermittelten Wertebereichen über Simulationen optimieren. Hierbei wird jeweils eine Antennenabmessung variiert und die Auswirkung auf eine oder mehrere der angegebenen Eigenschaften analysiert. Die Abb. 3 zeigt das Ergebnis einer Bandbreitenoptimierung für das GSM-

\footnotetext{
${ }^{2}$ Der Transient Solver nutzt die Finite Integration Technique zur Lösung der Maxwell-Gleichungen.
}

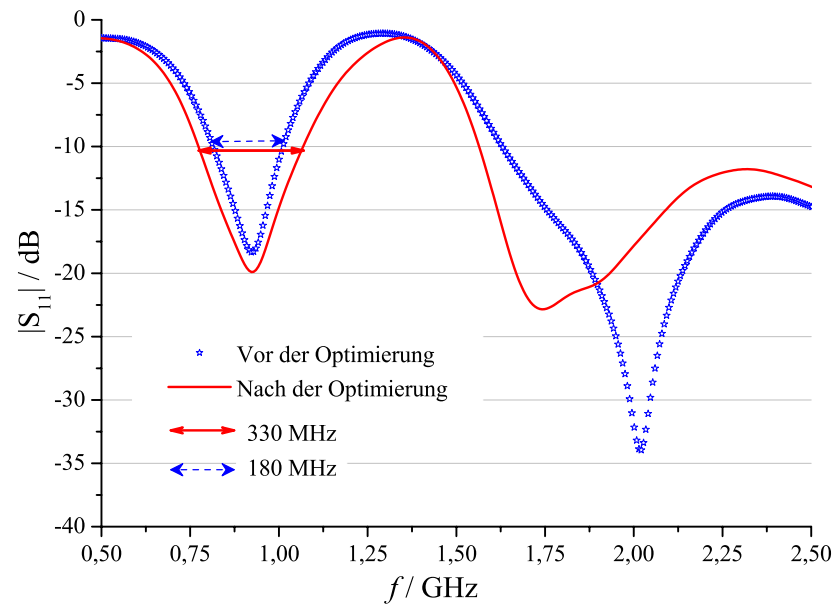

Abbildung 3. Bandbreiteoptimierung für das GSM-Band.

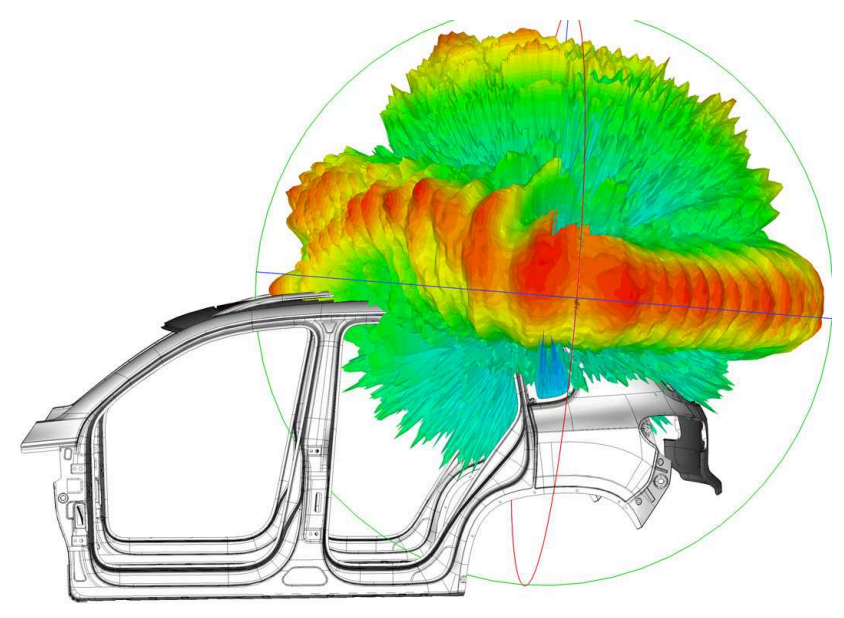

Abbildung 4. 3-D-Directivity-View aus der Multiband-Antenne-Simulation bei 5,9 GHz mit Panoramafenster im Autodach.

Band. Durch einen reduzierten Schlankheitsgrad wird die Bandbreite von $180 \mathrm{MHz}$ auf $330 \mathrm{MHz}$ erhöht. Somit kann das LTE- und GSM-Band mit einer gemeinsamen Antennenstruktur abgedeckt werden. Da die reale Antennenumgebung sowohl die S-Parameter als auch die Richtcharakteristik beeinflusst, wird bei der Simulation die Autokarosserie berücksichtigt (vgl. Abb. 4). Dadurch erhöht sich die Anzahl der Netzgitterelemente des Simulationsmodells auf 300 Millionen. Die Simulation der in Abb. 4 dargestellten Modell dauerte 23 Stunden und 2 Sekunde (24 Kerne, 48 GB Arbeitsspeicher). Einige Automodelle sind mit einem großen Panoramaglasdach ausgestattet. Die Glasscheibe beeinflusst aufgrund ihrer großen Fläche und ihrer hohen relativen Permittivität $\varepsilon_{\mathrm{r}}$ die Richtcharakteristik stark in Fahrtrichtung (vgl. Kwoczek, 2011 und Abb. 4). 


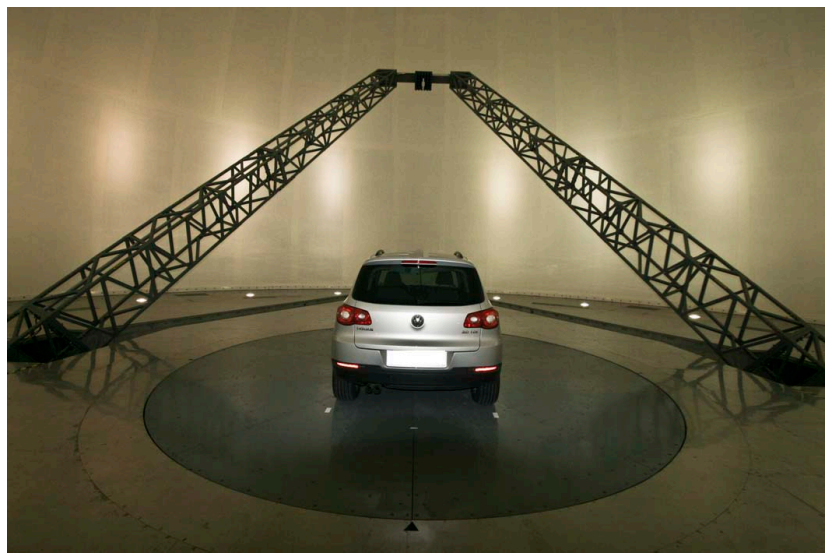

Abbildung 5. Messaufbau für eine Multiband-Antenne mit Fahrzeug.

\subsection{Fertigung}

Bei der Fertigung müssen die Eigenschaften der angewendeten Materialien berücksichtigt werden. Beispielsweise können einige Kunststoffe nicht über Klebeverfahren miteinander verbunden werden oder ausgewählte Materialien vertragen die hohe Temperatur während des Verlötens der elektronischen Komponenten nicht. Als Autodachantenne muss die Konstruktion zusätzlich die mechanischen Anforderungen, wie z. B. Wasserfestigkeit, geringes Gewicht sowie eine leichte und schnelle Montage erfüllen. Darüber hinaus ist eine industrielle Produzierbarkeit der Antennenkonstruktion für den Einsatz an Serienfahrzeugen erforderlich. Hierfür kann die Multiband-Antenne z. B. mit einer Kunststoffmetalliesierungstechnik kostengünstig hergestellt werden.

\subsection{Messung}

In diesem Schritt werden der Eingangsreflexionsfaktor $\left|S_{11}\right|$ und die 3-dimensionale Richtcharakteristik vermessen. Die $\left|S_{11}\right|$-Messung wird im Freiraum, die 3-D-Feldmessungen hingegen in einer Messhalle durchgeführt (vgl. Abb. 5). Die Messhalle besteht aus Kunststoff, der für elektromagnetische Wellen von $20 \mathrm{MHz}$ bis $8 \mathrm{GHz}$ transmittierend wirkt. Das Messsystem ist ein sphärisches Nahfeld-Messsystem von der Firma Nearfield Systems Inc. (NSI). Die Messsonde hängt an einem dielektrischen Hebelarm der Länge 11,5 m. Sie kann sich vertikal von $\vartheta=0^{\circ}$ bis $\vartheta=90^{\circ}$ bewegen. Das Fahrzeug befindet sich auf einer um $360^{\circ}$ drehbaren Bodenplatte. Bei der Messung befindet sich die Antennenebene 1,6 m über der Bodenplatte. Mit der Messanordnung kann die Richtcharakteristik des oberen Halbraums vermessen werden. Diese Messanordnung besitzt keinen Absorber. Die Reflexionen aus Bodenplatte, Hebelarm und Hallenwand werden durch einen sogenannten "Mathematical Absorber Reflection Suppression (MARS) technique" (Hindman and Newell, 2005) berücksichtigt. Die Nahfeld-Messergebnisse werden
Tabelle 2. Vermesse Frequenzen der Antenne.

\begin{tabular}{lllll}
\hline $\begin{array}{l}\text { LTE/GSM } \\
(\mathrm{MHz})\end{array}$ & $\begin{array}{l}\text { UMTS } \\
(\mathrm{MHz})\end{array}$ & $\begin{array}{l}\text { WLAN 2,4 } \\
(\mathrm{MHz})\end{array}$ & $\begin{array}{l}\text { WLAN 5,1 } \\
(\mathrm{MHz})\end{array}$ & $\begin{array}{l}\text { C2C } \\
(\mathrm{MHz})\end{array}$ \\
\hline 780 & 1710 & 2400 & 5150 & 5850 \\
810 & 1800 & 2500 & 5250 & 5880 \\
860 & 1900 & 2600 & 5350 & 5900 \\
960 & 2000 & 2690 & 5470 & 5925 \\
& 2100 & 3300 & 5600 & \\
& 2170 & 3800 & 5700 & \\
& & & 5825 & \\
\hline
\end{tabular}

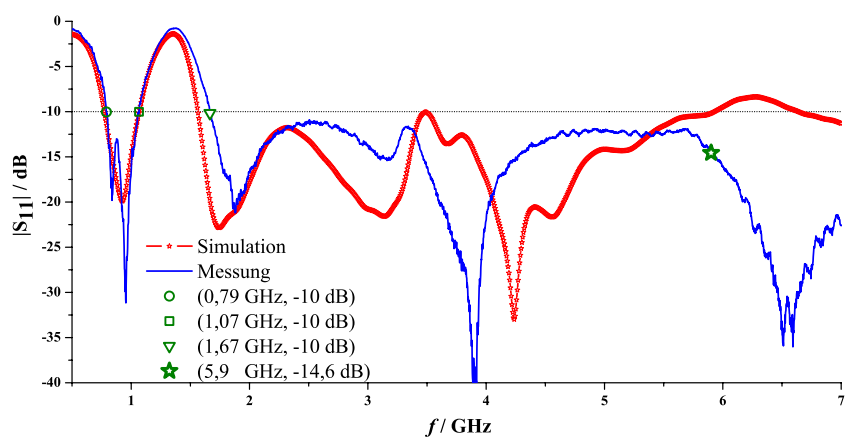

Abbildung 6. Vergleich der $\left|S_{11}\right|$-Verläufe der Simulation und der Messung.

nach der Messung durch Fourier-Transformation mit Hilfe der NSI 2000 Software $^{3}$ ins Fernfeld umgerechnet (Dan and Consultant, 1985). Die Tabelle 2 stellt die Frequenzen dar, bei welchen die Messungen in dem hier angegebenen Beispiel durchgeführt wurden. Da in dieser Arbeit als Schwerpunkt die Richtcharakteristik für das Car2Car-Band behandelt wird, werden im Folgenden die Antennendiagramme für $5,9 \mathrm{GHz}$ dargestellt. Die Abb. 6 zeigt den $\left|S_{11}\right|$-Verlauf (Eingangsreflexionsfaktor) der Simulation sowie der Messung. In den Frequenzbereichen, in denen die Impedanz angepasst sein soll, liegt die Kurve, wie in Abschnitt 2.4 gefordert, unterhalb $-10 \mathrm{~dB}$. Aufgrund der Toleranzen bei der Fertigung sowie der Abweichung der realen $\varepsilon_{\mathrm{r}}$-Werte von den idealen, entstehen Differenzen zwischen den Mess- und Simulationsergebnissen. Das Vertikaldiagramm bei $\varphi=0^{\circ}$ wird in der Abb. 7 dargestellt.

Der Pfeil zeigt in Fahrtrichtung. Aufgrund des Einflusses durch das Panoramafenster zeigt die Darstellung eine Abweichung von ca. $12 \mathrm{~dB}$ zwischen $\vartheta=90^{\circ}$ (Front) und $\vartheta=-90^{\circ}$ (Heck).

Diese Abweichung fällt bei der Messung mit vollständigem Metalldach deutlich geringer aus (vgl. Abb. 8). In beiden Diagrammen zeigen sich gute Übereinstimmungen zwischen den Simulationen und den Messungen. Der Einfluss der Dachreling ist in der Abb. 9 zu erkennen. Obwohl das

\footnotetext{
${ }^{3}$ Die NSI 2000 Software ist ein Teil vom NSI Messsystem.
} 


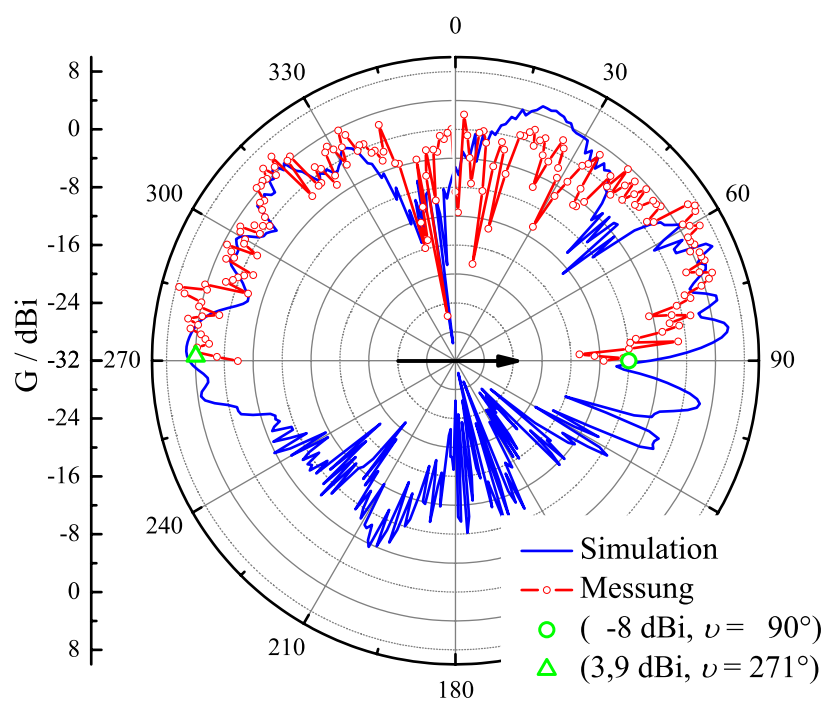

Abbildung 7. Vertikaldiagramm mit Panoramafenster $\left(\varphi=0^{\circ}\right)$.

Horizontaldiagramm zu den Fahrzeugseiten hin einen asymmetrisch schwankenden Verlauf aufweist, zeigt es zur Frontund Heckseite einen relativ hohen Antennengewinn mit nur kleinen Schwankungen. Bei einer Gegenüberstellung der entworfenen Multiband-Antenne mit einer Vergleichsantenne von einem Automobilzulieferer zeigt sich in weiten Teilen ein ähnliches Horizontaldiagramm (vgl. Abb. 10). Allerdings weist die entworfene Multiband-Antenne im Bereich von $150^{\circ}<\varphi<210^{\circ}$ eine wesentlich bessere Richtcharakteristik auf.

\section{7 Übertragungsreichweite der Multiband-Antenne}

Mit einer Berechnung gemäß ETSI wird die Übertragungsreichweite zwischen zwei Fahrzeugen, die in gleicher Richtung hintereinander fahren, ermittelt (ETSI, 2005). Hierfür wird angenommen, dass eine Übertragung bei $5,9 \mathrm{GHz}$ mit einer Bandbreite von $10 \mathrm{MHz}$ und einer QPSK-Modulation realisiert werden soll. Um eine Datenrate von $3 \mathrm{Mbit} / \mathrm{s}$ mit dem genannten System erreichen zu können, wird nach ETSI an der Empfangsantenne eine minimale Empfangsleistung von $-85 \mathrm{dBm}$ benötigt. Die Übertragungsstrecke ist vollständig in LOS (Line Of Sight).

Für die Car2Car-Kommunikation, die nah an der Erdoberfläche stattfindet, werden im Path-loss-Modell die ersten $15 \mathrm{~m}$ ab der Sendeantenne als Freiraum angenommen. Dieser Abstand wird mit $d_{0}$ bezeichnet. Bei einer Entfernung $d$ über $d_{0}$ hinaus wird ein Path-loss-Faktor von $n=2,7$ gewählt (ETSI). Gemäß des Link-Budgets ergibt sich die Empfangsleistung $P_{\mathrm{e}}$ in $\mathrm{dBm} \mathrm{zu}$ :

$P_{\mathrm{e}}=P_{\mathrm{s}(\max )}-G_{\mathrm{s}(\max )}+G_{\mathrm{s}}+G_{\mathrm{e}}+L$.

Hierbei beträgt die Empfangsleistung, wie bereits angegeben, $P_{\mathrm{e}}=-85 \mathrm{dBm}$. Die EU-Vorgabe (EU, 2008) schränkt

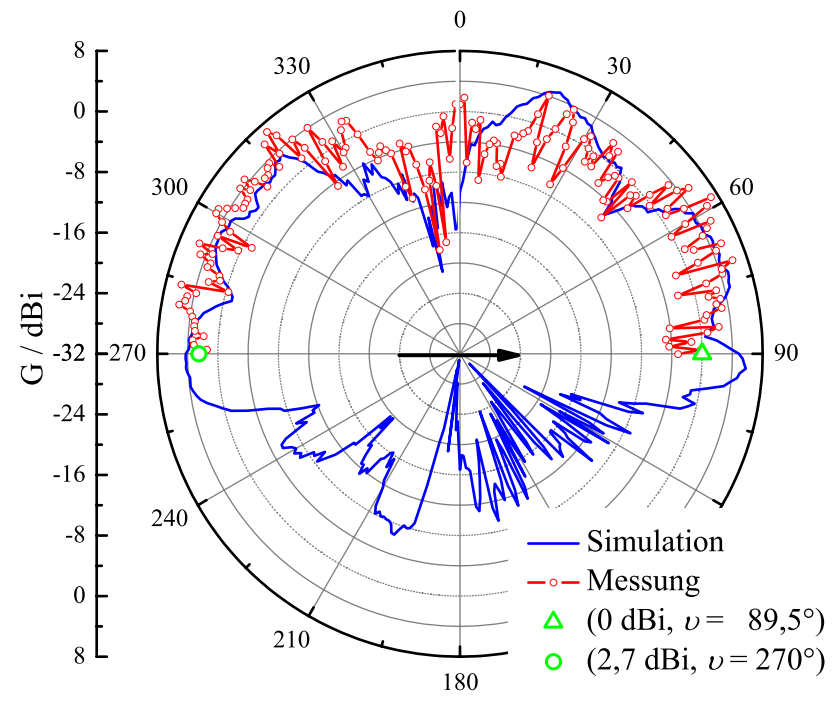

Abbildung 8. Vertikaldiagramm mit vollständigem Metalldach $\left(\varphi=0^{\circ}\right)$.

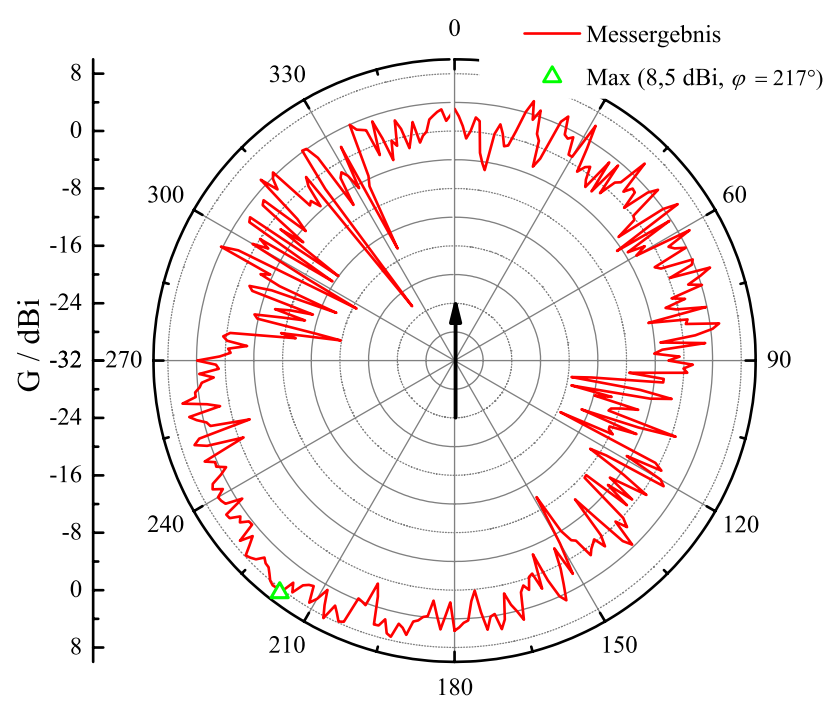

Abbildung 9. Horizontaldiagramm mit Dachreling $\left(\vartheta=90^{\circ}\right)$.

die maximale Sendeleistung auf $P_{\mathrm{s}(\max )}=33 \mathrm{dBm}$ (EIRP) ein. Der maximale Antennengewinn $G_{\mathrm{s}(\max )}=8,5 \mathrm{dBi}(\mathrm{vgl}$. Abb. 9) wird von der $P_{\mathrm{s}(\max )}$ abgezogen. $G_{\mathrm{s}}$ ist der Antennengewinn des Senders, welcher in Richtung Empfänger zeigt. Für die hier behandelte Antenne beträgt $G_{\mathrm{s}}=0,5 \mathrm{dBi}$ (Mittelwert von $-10^{\circ}<\varphi<10^{\circ}$ in Abb. 9). $G_{\mathrm{e}}$ ist der Antennengewinn des Empfängers, welcher in Richtung Sender zeigt. Für die hier behandelte Antenne beträgt $G_{\mathrm{e}}=4 \mathrm{dBi}$ (Mittelwert von $-170^{\circ}<\varphi<170^{\circ}$ in Abb. 9). Der Pfadverlust $L$ besteht aus zwei Anteilen. Zum einen aus dem Pfadverlust $L_{1}$ im Freiraum für die ersten $15 \mathrm{~m}$ und zum anderen aus dem Pfadverlust $L_{2}$ mit dem Einfluss der Erdoberfläche. Der gesamte Pfadverlust $L$ berechnet sich in $\mathrm{dB}$ zu: 


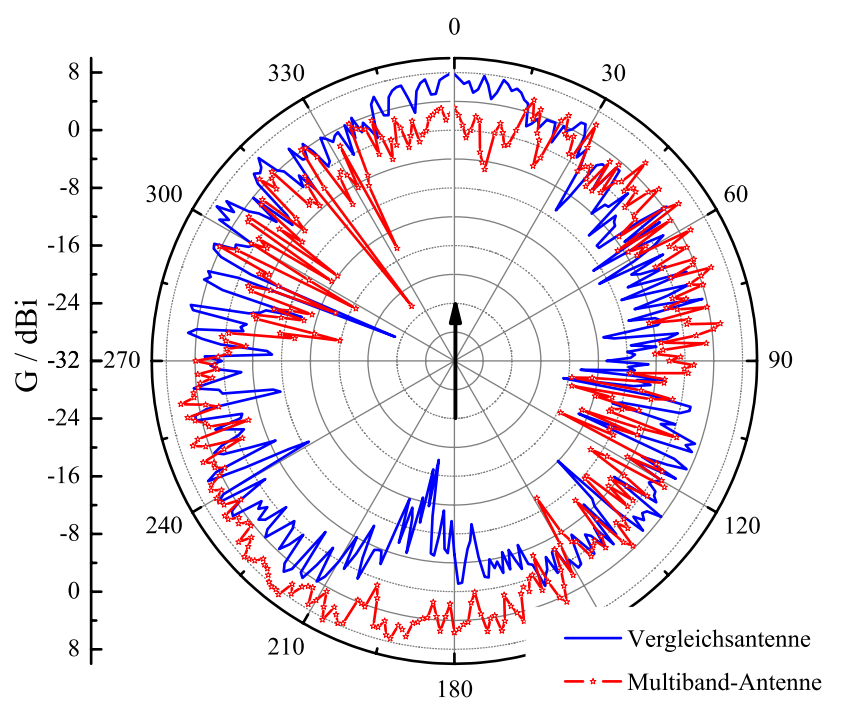

Abbildung 10. Horizontaldiagramme der Multiband-Antenne und der Vergleichsantenne $\left(\vartheta=90^{\circ}\right)$.

$L=L_{1}+L_{2}=20 \log \left(\frac{\lambda}{4 \pi d_{0}}\right)+10 \log \left(\frac{d_{0}}{d}\right)^{n}$.

Mit einer Wellenlänge $\lambda=5,08 \mathrm{~cm}$ sowie Gl. (1) und Gl. (2) folgt eine Übertragungsreichweite von

$d+d_{0}=568+15=583 \mathrm{~m}$.

\section{Zusammenfassung}

Mit dem vorgestellten Entwurfskonzept ist eine MultibandAntenne entworfen worden. Mit Messergebnissen konnten die Zielsetzungen verifiziert werden. Hierzu zählen die Frequenzabdeckung von $780 \mathrm{MHz}$ bis $5,925 \mathrm{GHz}$ und die maximale Antennenhöhe von $70 \mathrm{~mm}$. Es zeigt sich, dass die Multiband-Antenne für eine Car2Car-Anwendung keine benachteiligte Richtung auf dem Horizontaldiagramm enthält.

\section{Literatur}

Balanis, C. A.: Antenna Theory Analysis and Design, 3. Edn., John Wiley and Sons, Inc., USA, 2005.

Dan, S. and Consultant, A.: Near-Field Test Facility Design Antenna Measurement Techniques Association Conference, TRW Space Communications Division, Antenna Laboratory, Redondo Beach, Canada, 1985

Europäische Union: Regelung Nr. 26 der Wirtschaftskommission der Vereinten Nationen für Europa (UN/ECE) - Einheitliche Bedingungen für die Genehmigung der Kraftfahrzeuge hinsichtlich ihrer vorstehenden Außenkanten, in: Amtsblatt der Europäischen Union, 37, 14 August 2010.

European Telecommunications Standards Institute (ETSI): Part 1 Technical characteristics for pan-European harmonized communications equipment operating in the $5 \mathrm{GHz}$ frequency range and intended for critical road-safety applications, France, ETSI TR 102 492-1 V1.1.1, 2005-2006.

Europäische Union: Entscheidung der Kommission zur harmonisierten Nutzung von Funkfrequenzen im Frequenzband 5875$5905 \mathrm{MHz}$ für sicherheitsbezogene Anwendungen intelligenter Verkehrssysteme (IVS), in: Amtsblatt der Europäischen Union, 24, 5 August 2008.

Hindman, G. and Newell, A. C.: Reflection suppression in large spherical near.field range, Nearfield Systems Inc., Torrance, Canada, 2005.

Kwoczek, A.: Influence of Car Panorama Glass Roofs for Car2Car Communication, in: European cooperation in the field of scientific and technical research (EURO-COST), COST IC1004 TD(11) 01020, Lund, Sweden, 2011.

Lange, K. and Löcherer, K.-H.: Taschenbuch der Hochfrequenztechnik Band 2: Komponenten, 5. Auflage, Springer, Germany, 1992.

Lübke, A.: Car-to-Car Communication - Technologische Herausforderungen, in: Fachtagungsbericht GMM, VDE-Verlag, VDEKongress 2004, 18-20 Oktober 2004, Berlin, Germany, 2004.

Rothammel, K. and Krischke, A.: Antennenbuch, 12. Auflage, DARC Verlag Baunatal, Germany, 2001 